\title{
Deamination of the Partially N-Deacetylated Acid Mucopolysaccharides
}

\author{
By \\ Zensaku Yosizawa \\ From the Department of Biochemistry, Tohoku University \\ School of Medicine, Sendai
}

(Received for publication, May 1, 1963)

Stacey et al. ${ }^{1,2)}$ reported that the hexosaminidic linkages in several glucosaminides, chitosan and $\psi$-heparin ( $\mathrm{N}$-desulfated heparin) were cleaved deaminatively to produce 2,5-anhydromannose as the main product with nitrous acid. And they claimed that the influence of the configuration of the glycosidic center in glucosaminides on their rate of deamination was of use in establishing the configuration of the glucosaminidic linkages in certain mucopolysaccharides. Subsequently, Matsushima and $\mathrm{Fujii}^{3}$ ) showed that N-deacetylated galactosaminidic bonds in the hydrazinolyzate of chondroitin sulfate were cleaved to yield 2,5-anhydrohexose by the deamination. However, Yosizawa and Sato ${ }^{4}$ noticed that a part of the $\mathrm{N}$-deacetylated hexosaminidic linkages in the partially $\mathrm{N}$ deacetylated blood-group A mucopolysaccharide of hog gastric mucus were not cleaved deaminatively to give rise to 2,5-anhydrohexoses with nitrous acid. Moreover, more than sixty per cent of the $\mathrm{N}$-deacetylated hexosamines in the hydrazinolyzates of sodium chondroitin sulfate $\mathrm{A}$ and sodium hyaluronate could not be determined as 2,5-anhydrohexoses by the direct deamination. ${ }^{5}$ )

In an attempt to verify the deamination reactions of the $\mathrm{N}$-deacetylated hexosamines in the hydrazinolyzed preparations of sodium chondroitin sulfate $\mathrm{A}$ and of sodium hyaluronate, the present author examined the deamination products of these partially $\mathrm{N}$-deacetylated acid mucopolysaccharides. The experimental results showed that $\mathrm{N}$-deacetylated hexosamines in these preparations were converted not only to 2,5-anhydrohexoses but also to hexoses by the direct deamination. And the amounts of hexoses found in the non-dialyzable matters of the deamination products were more than twice as much as those of 2,5-anhydrohexoses.

The present findings together with other observations indicate that the $\mathrm{C}_{3}$ substituted hexosaminidic linkages are, more or less, resistant against the

吉沢善作 
deaminative cleavage to form 2,5-anhydrohexoses with nitrous acid. Hence, the susceptibility of the deaminative cleavage of the hexosaminidic linkages may give a clue in establishing not only the hexosaminidic linkages ${ }^{1,2)}$ but also the position of the substitution on the hexosamines in certain mucopolysaccharides.

\section{MATERIALS AND METHODS}

Materials - Partially N-deacetylated preparations of sodium chondroitin sulfate $\mathrm{A}$ and of sodium hyaluronate were the hydrazinolyzed products described in the previous paper.5) The brief preparation procedures of these materials were as in the following. Sodium chondroitin sulfate $\mathrm{A}$ and sodium hyaluronate were separately heated in a sealed tube with excess anhydrous hydrazine in a boiling water bath for 10 hours. After hydrazine was removed, each reaction product was dissolved in water and dialyzed. The non-dialyzable fraction of the product was concentrated to a small volume, then the substance was precipitated with ethanol and dried.

Methods of Analysis - Hexosamines were analyzed by the procedure of Yosizawa $^{6}$ with Dische-Borenfreund reaction. ${ }^{7)}$ 2,5-Anhydrohexoses were determined colorimetrically after being heated directly with the indole-hydrochloric acid reagent of Dische and Borenfreund, $\left.{ }^{7}\right)$ in which the difference of the optical densities at $492 \mathrm{~m} \mu$ and $550 \mathrm{~m} \mu\left(\mathrm{D}_{492}-\mathrm{D}_{550}\right)$ was read against the blank. Standard 2,5-anhydrohexose was prepared from glucosamine hydrochloride by the deamination according to the procedure of hexosamine analysis. ${ }^{6)}$ Glucuronic acid was estimated by the method of Dische ${ }^{8)}$ with carbazole-sulfuric acid reagent. Hexoses were analyzed by the $\mathrm{HR}_{10}$ method of Masamune and Sakamoto ${ }^{9}$ ) with thionalide-sulfuric acid reagent, in which the difference of the optical densities at $382 \mathrm{~m} / \mu$ and $440 \mathrm{~m} \mu\left(\mathrm{D}_{\mathbf{3 8 2}}-\mathrm{D}_{\mathbf{4 4 0}}\right)$ was read against the blank. Since 2,5anhydromannose prepared by the procedure of Akiya and Osawa ${ }^{10}$ gave the same absorption spectrum as that of hexoses in the $\mathrm{HR}_{10}$ reaction, 2,5-anhydrohexose correction was made together with glucuronic acid correction in the hexose analysis.

Paper chromatography of non-amino sugars - Paper chromatography of sugars was carried out on Toyo Roshi filter paper No. 3, in the descending way by the procedure of Masamune and Yosizawa ${ }^{11)}$ using solvent mixtures of n-butylacetateacetic acid-butanol-methanol-water $(3: 2: 2: 1: 1 \text {, by volume })^{11)}$ and of ethylacetate-pyridine-water $(2: 1: 2$, by volume, upper layer $) .^{12}$ Anilinehydrogen-phthalate was used as an indicator.

\section{RESULTS}

An aqueous solution $(5 \mathrm{mg}$ in $10 \mathrm{ml}$ ) of each partially $\mathrm{N}$-deacetylated acid mucopolysaccharide (the hydrazinolyzed preparations of sodium chondroitin sulfate $A$ and of sodium hyaluronate) was deaminated by the addition of $30 \mathrm{ml}$ of $5 \%$ 
aqueous sodium nitrite and $30 \mathrm{ml}$ of $33 \%$ acetic acid, followed by the shaking of the mixture for 30 minutes at room temperature. The reaction mixture was dialyzed in a visking casing against many changes of distilled water (51) for 5 days. The non-dialyzable fraction in the casing was concentrated to $50 \mathrm{ml}$ under reduced pressure. The concentrates obtained from the deamination products of the partially $\mathrm{N}$-deacetylated preparations of sodium chondroitin sulfate $\mathrm{A}$ and of sodium hyaluronate were designated as $\mathrm{CD}$ and $\mathrm{HD}$, respectively.

Each $40 \mathrm{ml}$ of the concentrates (CD and HD) was evaporated to dryness under reduced pressure. To each residue was added $4 \mathrm{ml}$ of $1 \mathrm{~N}$ sulfuric acid, then the solution was heated in a stoppered-glass tube for 10 hours in a boiling water bath. Sulfuric acid in each hydrolyzate was removed with barium carbonate and the resultant each filtrate was separately passed through a column $(2 \times 15 \mathrm{~cm})$

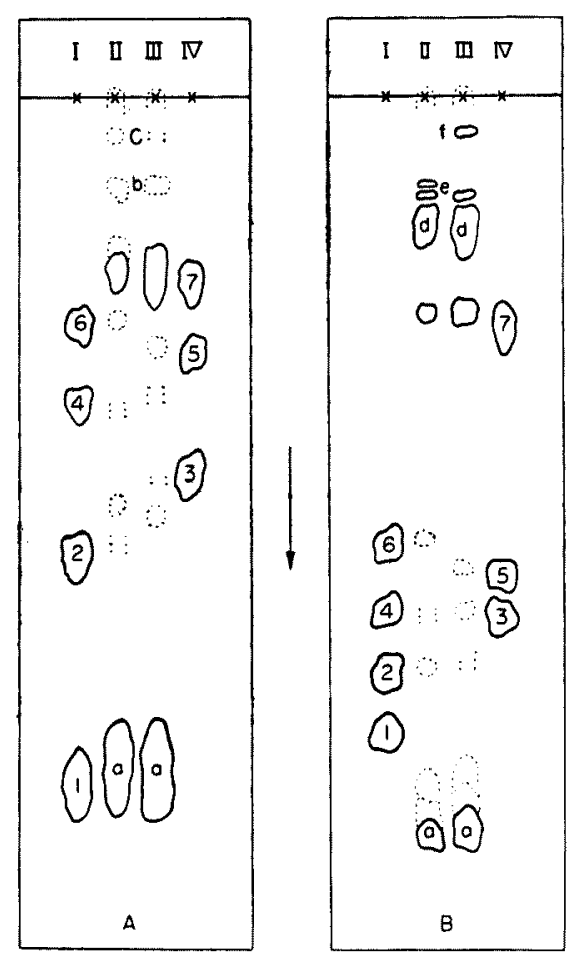

Fig. 1. Paper chromatograms of the non-amino sugars in the non-dialyzable matters of the deamination products of the partially $\mathbf{N}$-deacetylated preparations of sodium chondroitin sulfate $A(I T)$ and of sodium hyaluronate (III).

A: Developed with n-butylacetate-acetic acid-butanol-methanol-water $(3: 2: 2: 1: 1)$ for 33 hours at $28-30^{\circ} \mathrm{C}$. B: Developed with ethylacetate-pyridine-water $(2: 1: 2$, upper layer) for 16 hours at $28^{\circ}-30^{\circ} \mathrm{C}$. I \& IV: Authentic samples. 1: Rhamnose. 2: Lyxose. 3: Arabinose. 4: Mannose. 5: Glucose. 6: Galactose. 7: Glucuronic acid. II \& III: Test samples. a: Glucuronolactone. b,c,d,e,f: Glucuronic acid-containing compounds. 
of Amberlite IR-120 $\left(\mathrm{H}^{+}\right)$, followed by washing the column with water $(500 \mathrm{ml})$. The effluent and washing of each column were concentrated together to a small volume under reduced pressure. The non-amino sugars in each concentrate were checked by the paper chromatography.

The paper chromatograms, shown in Fig. 1, revealed that the non-dialyzable matters in the deamination products of the partially $\mathrm{N}$-deacetylated acid mucopolysaccharides contained large amounts of glucuronic acid, glucuronolactone and glucuronic acid-containing substances and small amounts of pentoses. In addition, the spots indicating small amounts of hexoses (galactose from $\mathbf{C D}$, glucose from HD) were also detected on the paper chromatograms.

On the other hand, sugar components in the non-dialyzable matters of the deamination products (CD and HD) were analyzed. The analytical data are shown in TABLES I \& II.

TABLE I. Analytical Data on the Non-dialyzable Matter (CD)

in the Deamination Product of the Partially N-Deacetylated Sodium Chondroitin Sulfate A

\begin{tabular}{l|c|c}
\hline \multicolumn{1}{c|}{ Analysis } & Material (\%) & Product (\%)* \\
\hline Glucuronic acid & 39.2 & 29.9 \\
Galactosamine & $32.4(3.4)^{* *}$ & 20.8 \\
2,5-Anhydrohexose & 0.1 & 2.3 \\
Hexose (as galactose) & - & 5.1
\end{tabular}

* The values were calculated on the basis of the amounts of the material used in the experiments.

** The value in parenthesis showed the amount of hexosamine, which could be converted to 2,5-anhydrohexose by the deaminative cleavage of the hexosaminidic linkages by the direct deamination with nitrous acid.

Table II. Analytical Data on the Non-dialyzable Matter (HD) in the Deamination Product of the Partially N. Deacetylated Sodium Hyaluronate

\begin{tabular}{l|c|c}
\hline \multicolumn{1}{c|}{ Analysis } & Material (\%) & Product $(\%)^{*}$ \\
\hline Glucuronic acid & 49.0 & 43.0 \\
Glucosamine & $41.0(3.3)^{* *}$ & 30.9 \\
2,5-Anhydrohexose & 0.1 & 2.1 \\
Hexose (as glucose) & - & 5.7 \\
\multicolumn{2}{|c}{ *, ** Signify the same as in TABLE I. }
\end{tabular}

The analytical data showed that the $\mathrm{N}$-deacetylated hexosamines in the partially $\mathrm{N}$-deacetylated acid mucopolysaccharides were converted not only to 2,5-anhydrohexoses but also to hexoses by the direct deamination with nitrous 
acid. The amounts of hexoses found in the non-dialyzable matters of the deamination products were more than twice as much as those of 2,5-anhydrohexoses.

\section{DISCUSSION}

As reported in the previous paper, ${ }^{5)}$ the per cent $\mathrm{N}$-deacetylation of $\mathrm{N}$ acetylgalactosamine and $\mathrm{N}$-acetylglucosamine in the hydrazinolyzed preparations of sodium chondroitin sulfate $\mathrm{A}$ and of sodium hyaluronate, used in the present experiments, were 28.2 per cent and 22.9 per cent, respectively. Moreover, the per cent contents of the hexosamine, which could be determined as 2,5-anhydrohexose by the direct deamination with nitrous acid, in total hexosamine in these materials were 10.5 per cent and 8.0 per cent, respectively. These data revealed that approximately $37 \sim 35$ per cent of the $\mathrm{N}$-deacetylated hexosaminidic linkages were cleaved deaminatively to give rise to 2,5 -anhydrohexoses with nitrous acid.

The present experiments showed that $\mathrm{N}$-deacetylated hexosamines in the partially $\mathrm{N}$-deacetylated acid mucopolysaccharides were converted not only to 2,5anhydrohexoses but also to hexoses by the deamination. Since the amounts of hexoses found in the non-dialyzable matters of the deamination products were more than twice as much as those of 2,5-anhydrohexoses, it was inferred that the alteration of amino radical to hydroxyl group was more predominant than the eliminative cleavage of the hexosaminidic linkages to form 2,5-anhydrohexoses in the deamination reactions of the present materials.

On the other hand, Stacey et al..$^{1,2)}$ and Matsushima and Fujïi) reported that the hexosaminidic linkages in several glucosaminides, chitosan, $\psi$-heparin and $\mathrm{N}$-deacetylated chondroitin sulfate were cleaved to form 2,5-anhydrohexoses as the main products by the deamination. Although it was elucidated that $\beta$-glucosaminidic linkages were cleaved more effectively than $\alpha$-glucosaminidic linkages with nitrous acid, ${ }^{1,2)}$ these authors did not give any quantitative data as to the formation of 2,5-anhydrohexoses by the deamination of these substances.

As mentioned already, we noticed that a part of the $\mathrm{N}$-deacetylated hexosaminidic linkages in the partially $\mathrm{N}$-deacetylated blood-group $\mathrm{A}$ mucopolysaccharide of hog gastric mucus were not cleaved deaminatively to yield 2,5-anhydrohexoses with nitrous acid. In case of $\psi$-heparin, the $\mathrm{N}$-desulfated glucosamine in the polysaccharide could be determined quantitatively as 2,5-anhydromannose by the direct deamination. ${ }^{\mathbf{1 3}}$

Chondroitin sulfate $A$ and hyaluronate are well known to contain the $\mathrm{C}_{3^{-}}$substituted $\beta$-hexosaminidic linkages. Recently, the linkage between glucosamine and glucuronic acid in heparin was shown to be glucosamine-4- $\alpha$ glucuronide. ${ }^{14)}$ On the other hand, the blood-group A mucopolysaccharide was revealed to have the $\mathrm{C}_{4}$-substituted hexosamine ${ }^{15-17)}$ and the $\mathrm{C}_{3}$-substituted 
hexosamine was also inferred to present in the substance, since human group-A substance contained both $\mathrm{C}_{3^{-}}$and $\mathrm{C}_{4}$-substituted hexosamines. ${ }^{18-22}$ )

The above observations, therefore, indicate that the $\mathrm{C}_{\mathbf{3}}$-substituted hexosaminidic linkages are, more or less, resistant against the deaminative cleavage to form 2,5-anhydrohexoses with nitrous acid. Hence, the susceptibility of the deaminative cleavage of the hexosaminidic linkages may give a clue in establishing not only the hexosaminidic linkages ${ }^{1,2)}$ but also the position of the substitution on the hexosamines in certain mucopolysaccharides.

\section{SUMMARY}

1. The partially $\mathrm{N}$-deacetylated preparations of sodium chondroitin sulfate A and of sodium hyaluronate were deaminated with nitrous acid.

2. The deamination products of the $\mathrm{N}$-deacetylated hexosamines in these partially $\mathrm{N}$-deacetylated mucopolysaccharides were shown to be hexoses and 2, 5 anhydrohexoses. The amounts of hexoses found in the non-dialyzable matters of the deamination products were more than twice as much as those of 2,5anhydrohexoses.

3. The experimental results indicate that the $\mathrm{C}_{3}$-substituted hexosaminidic linkages are, more or less, resistant against the deaminative cleavage to form 2,5anhydrohexoses with nitrous acid.

\section{References}

1) Foster, A.B., Martlew, E.F. \& Stacey, M., Chem. and Ind., 1953, 825.

2) Stacey, M., Ciba Foundation Symp. on the Chemistry and Biology of Mucopolysaccharides, London: Churchill Ltd., 1958, p. 4.

3) Matsushima, Y. \& Fujii, N., Bull. Chem. Soc. Japan, 1957, 30, 48.

4) Yosizawa, Z. \& Sato, T., J. Biochem., 1962, 51, 233.

5) Yosizawa, Z. \& Sato, T., J. Biochem., 1962, 51, 155.

6) Yosizawa, Z., Tohoku J. Exper. Med., 1961, 74, 69.

7) Dische, Z. \& Borenfreund, E., J. Biol. Chem., 1950, 184, 517.

8) Dische, Z., J. Biol. Chem., 1947, 167, 189.

9) Masamune, H. \& Sakamoto, M., Tohoku J. Exper. Med., 1956, 63, 345.

10) Akiya, S. \& Osawa, T., J. Pharm. Soc. Japan, 1954, 74, 1259.

11) Masamune, H. \& Yosizawa, Z., Tohoku J. Exper. Med., 1953, 59, 1.

12) Kuhn, R., Gauhe, A. \& Baer, H.H., Chem. Ber., 1954, 87, 289.

13) Yosizawa, Z., unpublished data.

14) Wolfrom, M.L., Vercellotti, J.R. \& Horton, D., J. Org. Chem., 1962, 27, 705.

15) Yosizawa, Z., Tohoku J. Exper. Med., 1949, 51, 51.

16) Masamune, H. \& Yosizawa, Z., Tohoku J. Exper. Med., 1956, 64, 267.

17) Masamune, H., Yosizawa, Z. \& Haga, M., Tohoku J. Exper. Med., 1956, 64, 257.

18) Côté, R.H. \& Morgan, W.T.J., Nature, London, 1956, 178, 1171.

19) Morgan, W.T.J., Ciba Foundation Symp. on Biochemistry of Human Genetics, London, Churchill Ltd., 1959, p. 194.

20) Morgan, W.T.J., Proc. Royal Soc., B, 1960, 151, 308.

21) Cheese, I.A.F.L. \& Morgan, W.T.J., Nature, London, 1961, 191, 149.

22) Schiffman, G., Kabat, E.A. \& Leskowitz, S., J. Am. Chem. Soc., 1962, 84, 73. 